\title{
Intratympanic Contrast in the Evaluation of Menière Disease: Understanding the Limits
}

\author{
J. Bykowski, J.P. Harris, M. Miller, J. Du, and M.F. Mafee
}

\begin{abstract}
BACKGROUND AND PURPOSE: Studies describing endolymphatic hydrops in Menière disease after off-label intratympanic gadoliniumbased contrast have been limited by long acquisition times. We aimed to demonstrate the feasibility of post-intratympanic imaging on a 3T MR imaging system within a clinically tolerable acquisition time and to address potential pitfalls in acquisition or interpretation.
\end{abstract}

MATERIALS AND METHODS: FDA Investigational New Drug 115,342 and institutional review board approval were obtained for intratympanic injection of 8-fold diluted Gd-DTPA into the more symptomatic ear of 6 adults with Menière disease. 3T MR imaging was performed using a 3-inch surface coil before and up to 28 hours after injection using FLAIR to define the nonenhancing endolymphatic space within the enhancing perilymph. Variable FLAIR TI images were used to determine the impact of fluid-suppression on interpretation. Image quality was assessed for perilymphatic and extralabyrinthine contrast enhancement, definition of endolymphatic anatomy, and other anatomic variants or pathologic findings.

RESULTS: The surface coil afforded $0.375 \times 0.375 \mathrm{~mm}$ in-plane FLAIR resolution in $<4$ minutes 30 seconds, sufficient to perceive the nonenhancing spiral lamina, interscalar septa, and endolymphatic structures. Coronal views highlighted a potential interpretation pitfall of vestibular endolymphatic distention overestimation due to partial volume averaging. Varying FLAIR TI resulted in visible changes in the perception of the cochlear endolymphatic space. CSF enhancement was detectable at the internal auditory canal fundus on the injected side in half of the patients, which may confound interpretation.

CONCLUSIONS: Using a surface coil preserves high resolution within a clinically acceptable acquisition time. Pitfalls remain regarding the interpretation of these images and optimizing protocols across platforms in the absence of a clear internal reference for standardization.

ABBREVIATIONS: IAC = internal auditory canal; GBCA = gadolinium-based contrast agents; IT = intratympanic

M enière disease can be debilitating due to the severity and unpredictability of symptom onset. ${ }^{1}$ Currently, routine MR imaging plays a supportive role in the evaluation of these patients, mainly by excluding alternative diagnoses. The translation of in-

\footnotetext{
Received September 15, 2014; accepted after revision December 28.

From the Departments of Radiology (J.B., M.F.M., J.D.) and Otolaryngology (J.P.H.), University of California, San Diego Health System, San Diego, California; and Department of Otolaryngology (M.M.), University of California, San Francisco Medical Center, San Francisco, California.

This work was supported by the William Hanafee Award, American Society of Head and Neck Radiology.

Paper previously presented in part at: Annual Meeting of the American Society of Head and Neck Radiology, October 3-7, 2012; Miami Beach, Florida; and American Society of Neuroradiology Annual Meeting and the Foundation of the ASNR Symposium, May 17-22, 2014; Montreal, Quebec, Canada.

Please address correspondence to Julie Bykowski, MD, UC San Diego Health System, Department of Radiology, 410 Dickinson St, MC 8749, San Diego, CA 92103;

e-mail: jbykowski@ucsd.edu

http://dx.doi.org/10.3174/ajnr.A4277
}

tratympanic (IT) gadolinium-based contrast agents (GBCA) in animal models of endolymphatic hydrops ${ }^{2-6}$ into the evaluation of membranous labyrinthine spaces in human patients with Menière disease was pioneered by Nakashima et al. ${ }^{7-9}$ Following IT injection, contrast diffuses through the round window membrane and also likely though the oval window, resulting in perilymphatic space enhancement, while the endolymphatic space remains nonenhancing. The underlying premise is that increased production or impaired clearance of endolymph from the scala media results in deflection of the imaging-occult Reissner membrane into the perilymphatic space of the scala vestibuli.

Studies of IT injections at other centers have demonstrated that the endolymphatic space is conspicuously distended in patients with Menière disease and less commonly in other causes of sensorineural hearing loss. ${ }^{10,11}$ Most interesting, endolymphatic distention characterized by these methods has been demonstrated in $20 \%-65 \%$ of contralateral asymptomatic ears in patients with 
Table 1: Participant demographics

\begin{tabular}{|c|c|c|c|c|c|c|c|c|}
\hline \multirow[b]{2}{*}{ No. } & \multirow{2}{*}{$\begin{array}{l}\text { Age } \\
\text { (yr) }\end{array}$} & \multirow{2}{*}{$\begin{array}{c}\text { Sex, } \\
\text { Ethnicity }\end{array}$} & \multirow[b]{2}{*}{ Side } & \multirow{2}{*}{$\begin{array}{l}\text { Years } \\
\text { since } \\
\text { Onset }\end{array}$} & \multirow[b]{2}{*}{ Prior Therapy } & \multirow[b]{2}{*}{ Symptoms } & \multicolumn{2}{|c|}{ SRT/SDS ${ }^{a}$} \\
\hline & & & & & & & Right & Left \\
\hline 1 & 62 & Female & Left & 6 & HCTZ & $\sim 2$ Vertigo episodes/year & $20 \mathrm{~dB}$ & $90 \mathrm{~dB}$ \\
\hline & & & & & & & $96 \%$ at $60 \mathrm{~dB}$ & $4 \%$ at $100 \mathrm{~dB}$ \\
\hline 2 & 45 & Male & Right & 3 & HCTZ, oral steroids & $\begin{array}{l}\text { Waxing/waning roaring tinnitus, } \\
\text { occasional vertigo }\end{array}$ & $\begin{array}{l}15 \mathrm{~dB} \\
96 \% \text { at } 75 \mathrm{~dB}\end{array}$ & $\begin{array}{l}5 \mathrm{~dB} \\
100 \% \text { at } 45 \mathrm{~dB}\end{array}$ \\
\hline 3 & 55 & Male & Right & 1 & HCTZ & $\begin{array}{l}\text { Tinnitus } \times 1 \text { year, } \\
\text { episodic vertigo } \times 2 \mathrm{mo}\end{array}$ & $\begin{array}{l}15 \mathrm{~dB} \\
96 \% \text { at } 55 \mathrm{~dB}\end{array}$ & $\begin{array}{l}20 \mathrm{~dB} \\
96 \% \text { at } 80 \mathrm{~dB}\end{array}$ \\
\hline 4 & 63 & Female & Right & $>10$ & $\begin{array}{l}\text { Bilateral IT steroid injection, } \\
\text { oral steroid, HCTZ }\end{array}$ & $\begin{array}{l}8 \text { Vertigo attacks within } \\
2 \text { mo }\end{array}$ & $\begin{array}{l}65 \mathrm{~dB} \\
56 \% \text { at } 80 \mathrm{~dB}\end{array}$ & $\begin{array}{l}25 \mathrm{~dB} \\
96 \% \text { at } 50 \mathrm{~dB}\end{array}$ \\
\hline 5 & 53 & Female & Left & $>10$ & $\begin{array}{l}\text { Endolymphatic shunt, IT gentamicin } \\
\times 9 \text {, IT steroids, gent/dex } \\
\text { impregnated Gelfoam }{ }^{\text {b }} \text { sponge in } \\
\text { round window niche }\end{array}$ & Tinnitus and pressure & $\begin{array}{l}10 \mathrm{~dB} \\
96 \% \text { at } 45 \mathrm{~dB}\end{array}$ & $\begin{array}{l}30 \mathrm{~dB} \\
92 \% \text { at } 65 \mathrm{~dB}\end{array}$ \\
\hline 6 & 35 & Female & Right & 3 & HCTZ & $\begin{array}{l}\text { Episodic vertigo within prior } \\
2 \text { weeks }\end{array}$ & $\begin{array}{l}65 \mathrm{~dB} \\
44 \% \text { at } 80 \mathrm{~dB}\end{array}$ & $\begin{array}{l}10 \mathrm{~dB} \\
96 \% \text { at } 50 \mathrm{~dB}\end{array}$ \\
\hline
\end{tabular}

Note:-HCTZ indicates hydrochlorothiazide; SRT, speech reception threshold; SDS, speech discrimination score (\%) at supra-SRT level (decibel); gent/dex, gentamicin/dexamethasone.

${ }^{\mathrm{a}}$ Normal $=$ Less than 25 , mild $=26-40$, moderate $=41-55$, moderate $/$ severe $=56-70$, severe $=71-90$, profound hearing loss $>90$.

b Phadia, Uppsala, Sweden.

Menière disease via IT or IV-GBCA methods, ${ }^{12-14}$ furthering the debate regarding imaging overestimation or clinical underestimation of Menière disease.

A variety of sequences has been used to evaluate the ear following IT injection of GBCA, including 3D real inversion recovery, ${ }^{8,15} 3 \mathrm{D}$-inversion recovery TSE, ${ }^{16}$ and $3 \mathrm{D}$ sampling perfection with application-optimized contrast using different flip angle evolutions FLAIR, ${ }^{12}$ with versions of 3D FLAIR techniques most commonly used. ${ }^{7,17-19}$ While the availability of 32-channel head coils has improved resolution to $0.4 \times 0.4 \times 0.8 \mathrm{~mm}$, these volumetric sequences come at the cost of time, requiring up to 15 minutes for an acquisition, with potential for motion degradation and decreased SNR due to parallel imaging techniques. ${ }^{20,21}$ A recent publication has shown reduced acquisition time to 5 minutes 26 seconds but at the cost of resolution, achieving only $0.7 \times 0.7 \times 0.8 \mathrm{~mm}^{1{ }^{11}}$

The aims of our study were to optimize resolution of post-IT contrast images within a clinically reasonable acquisition time on a clinical 3T scanner and to address potential pitfalls in acquisition or interpretation that should be considered as research continues into this methodology.

\section{MATERIALS AND METHODS Participants}

A 39-year-old healthy, asymptomatic adult volunteer man was recruited for noncontrast MR imaging as part of sequence optimization. Six adult patients with clinically diagnosed Menière disease and symptoms refractory to therapy were recruited from our neurotology clinic. Demographics, symptoms, and audiometric findings are summarized in Table 1. Exclusions to participation included contraindications to MR imaging or GBCA. Diuretics were not stopped for this study. All 6 patients had recent ( $<6$ month prior) bilateral audiograms, and repeat audiometric evaluation of the symptomatic ear was performed within 1 week post-IT GBCA.

\section{IT Injection}

Under the auspices of FDA Investigational New Drug 115,342 and institutional review board approval, IT injection of the more symptomatic ear was performed by neurotology faculty or by neurotology fellows under direct faculty supervision. The external auditory canal was cleaned, the tympanic membrane of the more symptomatic ear was anesthetized with phenol, and a small vent hole was made by using a 23-ga spinal needle. A 1-mL syringe of 1:7 volume/volume Gd-DTPA (Magnevist; Bayer HealthCare Pharmaceuticals, Wayne, New Jersey) contrast from single-use vials diluted in sterile saline was hand-warmed to minimize caloric side effects. A total of $0.4-0.5 \mathrm{~mL}$ of the solution was injected into the tympanic cavity under direct visualization, until reflux occurred. The patient remained in a lateral decubitus position with the injected ear up for 30 minutes to facilitate contrast diffusion into the perilymphatic space. During this time, the patient was instructed to spit instead of swallowing saliva, to minimize drainage of tympanic contrast into the Eustachian tube.

\section{Imaging}

MR imaging was performed on a 3T TwinSpeed MR imaging system (Signa Excite HD; GE Healthcare, Milwaukee, Wisconsin) by using an 8-channel head coil and a 3-inch surface coil centered over the temporomandibular joint. The primary sequence for optimization was FLAIR, with T1 and FIESTA sequences then prescribed for anatomic correlation. Parameters used clinically with the 8-channel head coil were adapted to the reduced FOV of the 3 -inch surface coil while scanning the healthy volunteer subject with serial scans altering the matrix, number of excitations, section thickness, TR, and TI, with the goal of achieving at least 0.4 -mm in-plane resolution in $<5$-minute acquisition time. Because contrast to noise could not be easily assessed on the noncontrast FLAIR sequence, emphasis was placed on appropriate fluid suppression in the internal auditory canals and labyrinthine structures. Scan parameters are summarized in Table 1.

The 6 enrolled participants underwent imaging of the more symptomatic temporal bone by using the 3 -inch surface coil 20-28 hours after IT contrast injection, by using FLAIR, T1, and FIESTA sequences detailed in Table 1. The following variables were then introduced to assess the potential impact on scan schedule or interpretation: 1) Three participants also were 


\begin{tabular}{|c|c|c|c|c|c|c|c|c|c|c|c|}
\hline Coil & Sequence & $\begin{array}{c}\mathrm{TE} \\
\text { (ms) }\end{array}$ & $\begin{array}{c}\mathrm{TI} \\
\text { (ms) }\end{array}$ & $\begin{array}{c}\text { TR } \\
\text { (ms) }\end{array}$ & Matrix & $\begin{array}{l}\text { FOV } \\
(\mathrm{cm})\end{array}$ & $\begin{array}{c}\text { In-Plane } \\
\text { Resolution } \\
(\mathrm{mm})\end{array}$ & $\begin{array}{l}\text { Thickness } \\
\text { (mm) }\end{array}$ & $\begin{array}{l}\text { Flip } \\
\text { Angle }\end{array}$ & $\begin{array}{c}\text { Bandwidth } \\
(\mathrm{Hz})\end{array}$ & $\begin{array}{l}\text { Time to } \\
\text { Acquire } \\
\text { (min:s) }\end{array}$ \\
\hline \multirow[t]{3}{*}{ 8-Channel } & Cisternography (FIESTA) & 3 & & 6 & $320 \times 320$ & 18 & $0.56 \times 0.56$ & 1 & $55^{\circ}$ & 163 & $4: 27$ \\
\hline & 2D FLAIR & 122 & 2500 & 9454 & $320 \times 320$ & 18 & $0.56 \times 0.56$ & 2 & $90^{\circ}$ & 122 & 4:06 \\
\hline & T1 spin-echo ${ }^{b}$ & 9 & & 400 & $320 \times 320$ & 18 & $0.56 \times 0.56$ & 2 & $90^{\circ}$ & 163 & $4: 19$ \\
\hline \multirow[t]{4}{*}{ 3-Inch surface } & Cisternography (FIESTA) & 4 & & 8 & $320 \times 320$ & 12 & $0.38 \times 0.38$ & 1 & $55^{\circ}$ & 163 & $4: 35$ \\
\hline & 2D FLAIR (3 pt) & 124 & $2000^{c}$ & 10,000 & $320 \times 320$ & 12 & $0.38 \times 0.38$ & 2 & $90^{\circ}$ & 122 & 4:12 \\
\hline & 2D FLAIR (3 pt) & 123 & 2000 & 9000 & $320 \times 320$ & 12 & $0.38 \times 0.38$ & 2 & $90^{\circ}$ & 122 & $4: 24$ \\
\hline & T1 spin-echo ${ }^{b}$ & 11 & & 500 & $320 \times 320$ & 12 & $0.38 \times 0.38$ & 2 & $90^{\circ}$ & 163 & $5: 10$ \\
\hline
\end{tabular}

Note:-pt indicates patients

${ }^{\text {a }}$ Protocol $1(n=3)$, 1 scan session: $20-28$ hours post-IT contrast, includes pre- and post-IV contrast images. Protocol 2 ( $\left.n=2\right), 3$ scan sessions: 1) pre- and post-IV contrast images (no IT), 2) 2 hours post-IT contrast/4 hours post-IV contrast, 3) $20-28$ hours post-IT contrast/ 30 hours post-IV contrast. Protocol $2 B$ ( $n=1$; scheduling conflict precluded pre-IT injection imaging as above in protocol 2 number 1), 2 scan sessions: 1) 20 hours post-IT contrast with pre-and post-IV contrast images, 2) 25 hours post-IT contrast/4 hours post-IV contrast.

${ }^{\mathrm{b}}$ Parameters for T1 pre- and post-IV contrast scans did not change.

${ }^{c}$ FLAIR TI was varied from 1800 to $2800 \mathrm{~ms}$ for 2 patient scans, with all other parameters fixed.

scanned with the surface coil within 2 hours post-IT injection to assess the extent of contrast diffusion into the perilymph. 2) In 2 participants, FLAIR TI was varied from 2000 to $2800 \mathrm{~ms}$ during the 20- to 28-hour post-IT delayed surface coil scan session to assess the impact of variable fluid suppression on the interpretation of endolymphatic distention. 3) In 1 participant, dedicated coronal imaging was performed by using the surface coil FLAIR sequence for orthogonal evaluation; this was incomplete on a second patient due to a technical issue that ended the scanning session. Additionally, all 6 participants had imaging of the bilateral temporal bones with the 8-channel head coil by using FLAIR, FIESTA, and pre- and post-IV contrast T1 sequences (Table 1) to evaluate any regional anatomic variants or other pathology. In 2 patients, this was before any IT contrast; in 1 patient, it was within 2 hours after IT contrast injection (due to scheduling complications precluding pre-IT injection scanning); and in 3 patients, it occurred 20-28 hours after IT contrast injection.

\section{Assessment}

Participants were asked to describe any discomfort associated with the IT injection, IV injection, or scanning session (comfort, noise, and so forth). Image quality was assessed by 2 neuroradiologists with expertise in head and neck imaging (J.B., M.F.M.) to assess perilymphatic contrast enhancement, the definition of endolymphatic anatomy, any extralabyrinthine contrast enhancement, and other anatomic variants or pathologic findings. Apparent endolymphatic volume on delayed post-IT (20-28 hour) FLAIR images obtained with the surface coil was determined by visual assessment on the PACS workstation and compared with proposed grading scales. ${ }^{8,13}$

\section{RESULTS}

Four adult female and 2 adult male consenting adults with Menière symptoms refractory to medical therapy participated in this protocol (Table 2). All participants met the American Academy of Otolaryngology-Head and Neck Surgery 1995 criteria $^{22}$ of "Definite Meniere's Disease" with appropriate clinical imaging and audiometry. In-plane resolution of $0.375 \times 0.375 \mathrm{~mm}$ was achieved for the FLAIR sequence by using the 3 -inch surface coil, with a maximum acquisition time of 4 minutes 24 seconds. No scans were degraded by patient motion. There were no procedural complications from intratympanic injection or complications associated with the MR image. Patients reported mild discomfort at the time of phenol anesthesia of the tympanic membrane and a sense of aural fullness post-IT injection. Initially, we accepted audiogram results within the prior 6 months as a baseline. One participant demonstrated a change from mild to moderate lowfrequency hearing loss $(250-500 \mathrm{~Hz})$ on the post-IT injection audiogram compared with the baseline 5 months prior, with a speech reception threshold change from $15 \mathrm{~dB}, 96 \%$ speech discrimination score threshold, to $20 \mathrm{~dB}, 96 \%$ speech discrimination score. A repeat audiogram 6 weeks after IT contrast and 2 weeks after preplanned IT dexamethasone injection demonstrated a $10-\mathrm{dB}$ hearing improvement at $250 \mathrm{~Hz}, 15-\mathrm{dB}$ improvement at $500 \mathrm{~Hz}$, and speech reception threshold change of $15 \mathrm{~dB}, 96 \%$ speech discrimination score. The participant had ongoing symptoms since the baseline audiogram; however, we could not exclude any changes possibly attributed to contrast injection. At the patient's last audiogram approximately 11 months later, fluctuation was again noted, now with a speech reception threshold of 10 $\mathrm{dB}$, and a $100 \%$ speech discrimination score. Therefore, we revised the protocol to perform audiometry immediately before IT injection. No other audiometric changes between pre- and postinjection were identified in the 3 subsequent participants.

Assessment of surface coil FLAIR imaging 20-28 hours after IT injection showed contrast in the perilymphatic spaces of the basal, middle, and apical cochlear turns; vestibule; and semicircular canals of all injected ears (Fig 1), sufficient to visibly discriminate the nonenhancing spiral lamina, interscalar septa, and endolymphatic structures. In the 3 ears that were also imaged with the surface coil within 2 hours post-IT injection, contrast enhancement was only evident in the cochlear basal turn and vestibule. Contrast did not persist within the tympanic cavity in any delayed scans, though contrast was seen within mastoid air cells on the side of injection in 2 patients. Contrast was not evident in the perilymphatic space along the endolymphatic duct, and there was no endolymphatic duct or sac distention in any patient.

The nonenhancing endolymphatic space represented $34 \%$ $50 \%$ of the enhancing vestibule area in 4 ears and $>50 \%$ in 2 ears. This finding corresponds to mild and moderate vestibular hydrops, respectively, per the proposed Nagoya criteria, ${ }^{8}$ with only 

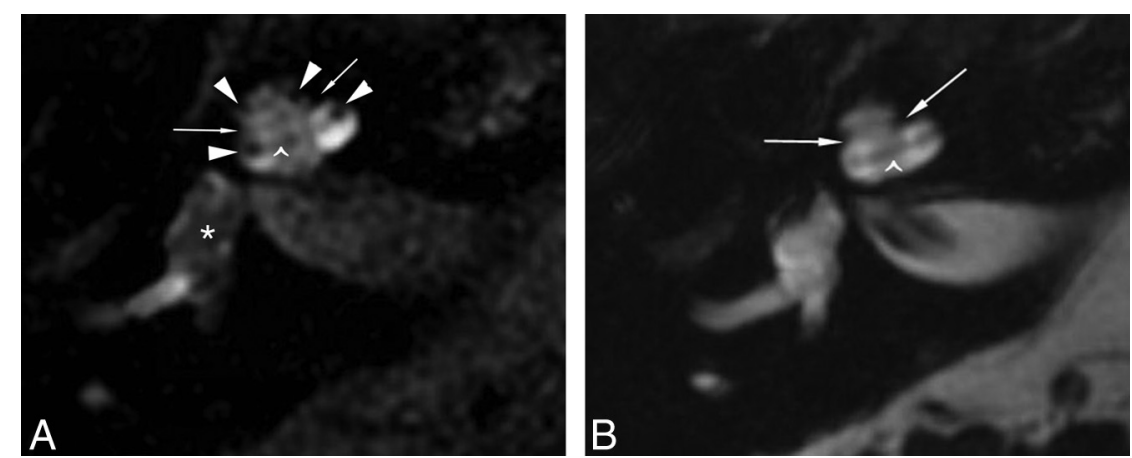

FIG 1. Differentiation of the perilymphatic-versus-endolymphatic spaces is evident when comparing FLAIR $(A)$ and FIESTA images $(B)$ obtained 21 hours after IT injection of 1:7 volume/volume diluted Magnevist contrast with a 3-inch surface coil. The nonenhancing fibro-osseous structures are evident on both sequences: the interscalar septa, separating the basal and middle turns and middle and apical turns (arrows), and the spiral lamina apparatus within each cochlear turn (caret). The FLAIR sequence shows central nonenhancement of the endolymphatic space of the vestibule ( $A$, asterisk) and suggestion of distention of the scala media ( $A$, arrowheads) into the scala vestibuli, whereas on FIESTA image $(B)$, the endolymph and perilymph are both hyperintense and indistinguishable (patient 2).
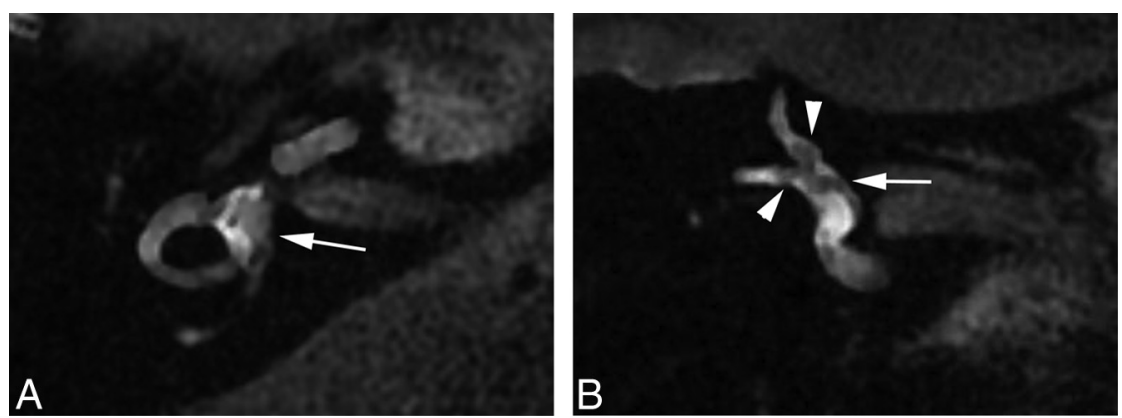

FIG 2. The nonenhancing endolymphatic space (A, arrow) occupies $>33 \%$ of the area of the vestibule on axial FLAIR image ( $A$ ) obtained 28 hours after IT GBCA injection, suggesting endolymphatic distention. Coronal FLAIR obtained concurrently $(B)$ demonstrates that the extent of distention of the endolymphatic space is overestimated on the axial view, due to partial volume averaging and section prescription through the membranous utricle ( $B$, arrow). Partial volume averaging also likely contributes to signal heterogeneity within the semicircular canals (A). Arrowheads $(B)$ correspond to the endolymphatic ductal ampullae of the superior and lateral semicircular canals (patient 6).
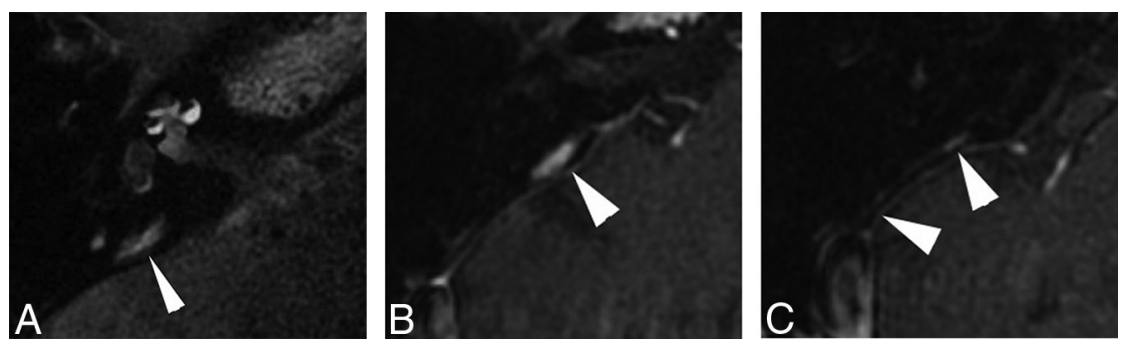

FIG 3. FLAIR imaging performed 28 hours after left IT contrast injection reveals a hyperintense structure $(A$, arrowhead) extending parallel to the expected course of the vestibular aqueduct (not seen). Comparison with positive-contrast T7-weighted images obtained before IT injection confirms that this structure is an enhancing dural vessel ( $B$ and $C$, arrowheads) coursing parallel to the posterior semicircular canal, extending from the middle cranial fossa to the sigmoid sinus. Correlation with anatomic imaging is imperative to avoid the misinterpretation of dilated endolymphatic space in the vestibular aqueduct (patient 4).

the 2 latter cases meeting grade I hydrops per Baráth et al. ${ }^{13}$ All surveyed ears demonstrated distention of the scala media without obliteration of the scala vestibuli, corresponding to mild cochlear hydrops ${ }^{9}$ and grade I hydrops ${ }^{13}$ respectively. Partial volume effects were evident when comparing axial and coronal planes, which could lead to overestimation of endolymphatic distention in the vestibule, depending on the section profile and thickness of axial images (Fig 2).

Variant vascular anatomy was noted in 3 patients, including a prominent vein paralleling the vestibular aqueduct on the symptomatic side (Fig 3), ICA hypoplasia contralateral to the symptomatic side, and a large, high-riding jugular bulb on the symptomatic side, without diverticulum.

If we kept all other parameters fixed, variation of the TI from 2000 to 2800 on surface coil FLAIR imaging 20-28 hours after IT injection resulted in a visually conspicuous difference of the nonenhancing endolymphatic space. Decreasing fluid suppression resulted in decreased conspicuity of the endolymphatic space in the cochlea at a longer TI (Fig 4).

Visual analysis of surface coil FLAIR and T1-weighted imaging 20-28 hours after IT injection revealed clear enhancement in the CSF at the internal auditory canal (IAC) fundus in 1 patient (Fig 5) and subtle enhancement in 2 additional patients. Enhancement was detectable on both FLAIR and T1 images and, therefore, was not attributed to incomplete fluid suppression; additionally, it was only evident at the fundus, not throughout the CSF of the IAC.

\section{DISCUSSION}

The diagnosis of Menière disease may be delayed for years in up to $20 \%$ of patients who ultimately receive the diagnosis. ${ }^{14}$ Since Mark et al ${ }^{23}$ reported asymmetric labyrinthine enhancement after IV contrast in patients with sudden deafness and vertigo, continued technologic advances have improved our ability to resolve the delicate structures of the inner ear. Different methods have been used to assess the membranous labyrinth, with inherent variations among scanner vendors, protocols, coils, and contrast agents. For this project, we focused on 1 promising option by using a 3-inch surface coil 20-28 hours after IT injection of diluted Magnevist. With an acquisition time of $<4$ minutes 30 seconds for the high-resolution FLAIR sequence, it could be easily added to a routine clinical IAC protocol, achieving better in-plane resolution within less time than any reported protocol to date.

Advantages of using the surface coil include the ability to re- 

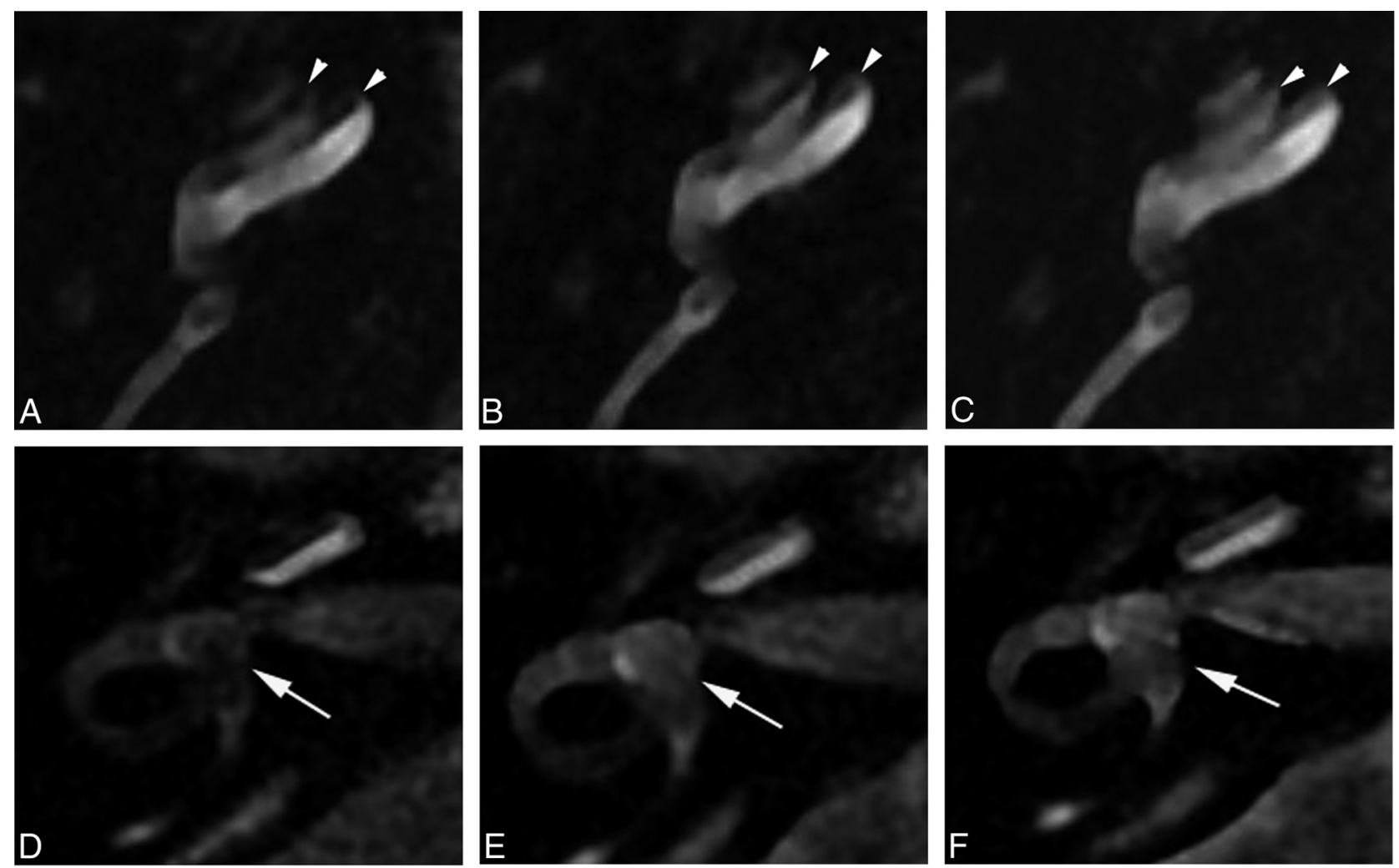

FIG 4. The impact of variable fluid suppression is visually evident with direct comparison of FLAIR sequences with Tls of $2000 \mathrm{~ms}$ ( $A$ and $D$ ), 2500 $\mathrm{ms}(B$ and $E)$, and $2800 \mathrm{~ms}(C$ and $F$ ), with all other parameters remaining fixed. The nonenhancing scala media (arrowheads) becomes less conspicuous during this short range of TI $(A-C)$, which may result in altered perception of endolymphatic space distention. Variation corresponding to the larger endolymphatic space in the vestibule (arrows, $D-F$ ) is less perceptible with changes in TI (patient 4).

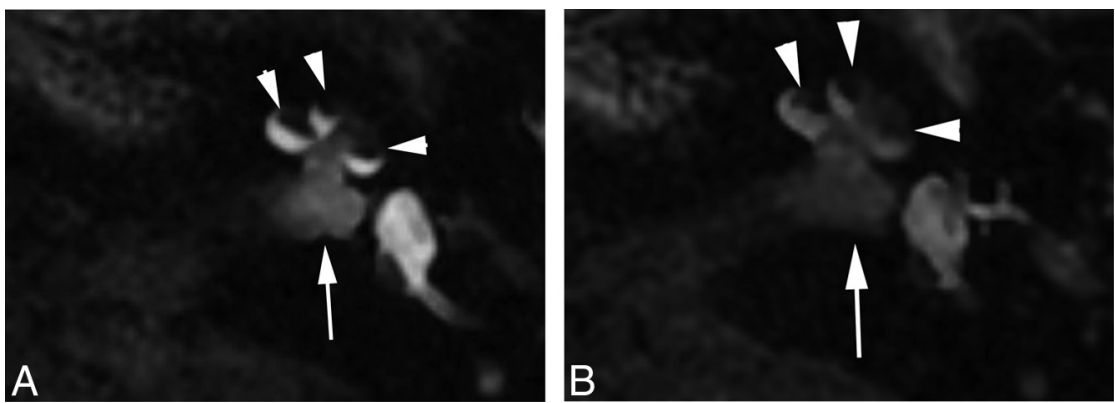

FIG 5. Enhancement is visible at the fundus of the IAC (arrow) on delayed imaging after IT GBCA, conspicuous on FLAIR $(A)$ and T1-weighted imaging $(B)$ in this patient who had profound distention of the endolymphatic space in the basal and middle cochlear turns (arrowheads) (patient 5). ing from 8- to 32-channel head coils, and techniques have been reproduced on a Philips Achieva 3T system (Best, the Netherlands). ${ }^{27}$ Because protocols vary across platforms and scanners, development of internal references/controls will be important for accurate characterization of findings. Our results demonstrate the visually conspicuous variation in the suppression of intrinsic endolymphatic fluid signal across a narrow range of TIs. While visual assessment can approximate the extent of fluid suppression through comparison to CSF signal duce the FOV with minimization of wrap artifacts due to the inherent limited penetration of the coil, reduced acquisition time compared with $3 \mathrm{D}$ techniques, and improved SNR relative to $3 \mathrm{D}$ sequences in which acceleration for parallel imaging results in signal loss. ${ }^{20,21}$ A limitation of the surface coil is that a $2-\mathrm{mm}$ section thickness was required for optimal signal, requiring precision in the prescription for centering the cochlea and vestibule. This can be overcome with the surface coil by obtaining direct coronal and oblique acquisitions. With the shorter acquisition time per scan, there is less risk of motion degradation that may occur with longer volumetric protocols. These techniques on a GE Healthcare 3T system have not been previously reported, though multiple sites have published series performed on Siemens Verio and Trio 3T scanners (Siemens, Erlangen, Germany), ${ }^{10,12,17,18,24-26}$ progress- in the IAC or cerebellopontine angle cistern, some method of assessment is important as these protocols are used, to ensure normalization and reproducibility before postprocessing algorithms are applied to the data. ${ }^{19,28}$

When one evaluates these protocols for the clinical setting, it is important to consider whether or how this information may change management. There remains interest in using IT GBCA as a surrogate for IT drug delivery, acknowledging differences in chemical composition and molecular weight among different GBCAs, steroids, and gentamicin. ${ }^{29}$ Poor-to-absent contrast penetration into the perilymph has been reported after IT injection in up to $18 \%$ of patients, ${ }^{9,30}$ suggesting a means to screen for potential poor responders to IT interventions. Nevertheless, series have not found a reliable correlation between the extent of en- 
dolymphatic distention and patient response to subsequent IT steroids, ${ }^{16,18}$ and there has been variable correlation between imaging with summating potential/action potential ratios electrocochleography and vestibular-evoked myogenic potential. ${ }^{17,25,31}$ These outcomes may be partially due to lack of standardization of criteria for severity based on imaging findings ${ }^{8,12,13}$ but also may reflect the spectrum of findings through the range associated with the natural history of Menière disease.

The delayed post-IT injection FLAIR sequence should be interpreted with multiplanar projections, to avoid interpretation errors due to partial volume averaging as illustrated in Fig 2. Additionally, any nonstandard sequences must be reviewed in the context of a full IAC protocol, including fluid-sensitive, DWI, FLAIR, and postcontrast T1 sequences of the entire auditory pathway, to exclude a schwannoma ${ }^{32}$ or other source of symptoms.

An unexpected finding in our study was the prevalence of enhancement in the CSF at the IAC fundus on 20- to 28-hour post-IT contrast FLAIR and T1 images. This was evident in half of our patients, and although our study group was small, this finding suggests that it may occur more frequently than has been noted in the literature. ${ }^{7,33,34}$ This observation prompts caution regarding proceeding directly to delayed postcontrast imaging in the evaluation of suspected Menière disease, to avoid potentially obscuring hemorrhage or protein that may be responsible for symptoms. ${ }^{10,35}$ MR imaging 4 hours after IV contrast has been promoted as an alternative, allowing simultaneous evaluation of both ears and not requiring IT injection or institutional review board approval. The 2 methods are complementary in that delayed IV methods reflect blood-labyrinthine barrier integrity, whereas IT methods reflect round window permeability; however, delayed IV methods have reduced contrast to noise. Careful attention to extralabyrinthine enhancement in both delayed IV and IT protocols is necessary for better understanding of the mechanism and significance of increased modiolar permeability and in the context of the timing of imaging for clinical interpretation. Despite the described limitations, the improved resolution and contrast achievable with IT methods should be of particular value as we continue to evaluate the reproducibility of these methods to define the normal and diseased endolymphatic systems.

\section{CONCLUSIONS}

Using a surface coil with a $3 \mathrm{~T}$ system affords high-resolution FLAIR imaging of the inner ear after IT contrast administration in a clinically reasonable time. Because these techniques are only an indirect evaluation of endolymphatic distention, however, and not a proved surrogate for Menière symptoms or response to therapy, continued investigation is necessary before use in clinical diagnosis. Evaluation should include correlation with a full IAC protocol to exclude other sources for symptoms, with awareness that diffusion of IT contrast into the CSF in the IAC may mimic or obscure hemorrhage or enhancement. Additionally, work remains to be done to optimize protocols in the absence of a clear internal reference for standardization and to better understand the implications of modiolar permeability.

\section{ACKNOWLEDGMENTS}

The authors would like to thank Sharon Mick, NP, for her help in coordinating patient scheduling.

Disclosures: Julie Bykowski-RELATED: Grant: American Society of Head and Neck Radiology Hanafee Award*; Other: Off-label use of Magnevist contrast per FDA Investigational New Drug 115,342. Mia Miller-UNRELATED: Grants/Grants Pending: Hearing Research Inc*; Travel/Accommodations/Meeting Expenses Unrelated to Activities Listed: resident travel grant, ${ }^{*}$ Mount Zion travel grant. ${ }^{*}$ Money paid to the institution.

\section{REFERENCES}

1. Committee on Hearing and Equilibrium guidelines for the diagnosis and evaluation of therapy in Meniere's disease: American Academy of Otolaryngology-Head and Neck Foundation, Inc. Otolaryngol Head Neck Surg 1995;113:181-85

2. Counter SA, Bjelke B, Borg E, et al. Magnetic resonance imaging of the membranous labyrinth during in vivo gadolinium (Gd-DTPABMA) uptake in the normal and lesioned cochlea. Neuroreport 2000;11:3979-83

3. Duan M, Bjelke B, Fridberger A, et al. Imaging of the guinea pig cochlea following round window gadolinium application. Neuroreport 2004;15:1927-30

4. Zou J, Pyykko I, Bjelke B, et al. Communication between the perilymphatic scalae and spiral ligament visualized by in vivo MRI. $A u$ diol Neurootol 2005; 10:145-52

5. Kakigi A, Nishimura M, Takeda T, et al. Effects of gadolinium injected into the middle ear on the stria vascularis. Acta Otolaryngol 2008;128:841-45

6. Zou J, Poe D, Bolje B, et al. Visualization of inner ear disorders with MRI in vivo: from animal models to human applications. Acta Otolaryngol Suppl 2009:22-31

7. Nakashima T, Naganawa S, Suguira M, et al. Visualization of endolymphatic hydrops in patients with Meniere's disease. Laryngoscope 2007;117:415-20

8. Nakashima T, Naganawa S, Pyykko I, et al. Grading of endolymphatic hydrops using magnetic resonance imaging. Acta Otolaryngol Suppl 2009:5-8

9. Nakashima T, Naganawa S, Katayama N, et al. Clinical significance of endolymphatic imaging after intratympanic gadolinium injection. Acta Otolaryngol Suppl 2009:9-14

10. Sugiura M, Naganawa S, Teranishi M, et al. Three-dimensional fluidattenuated inversion recovery magnetic resonance imaging findings in patients with sudden sensorineural hearing loss. Laryngoscope 2006;116:1451-54

11. Yamazaki M, Naganawa S, Tagaya M, et al. Comparison of contrast effect on the cochlear perilymph after intratympanic and intravenous gadolinium injection. AJNR Am J Neuroradiol 2012;33:773-78

12. Fang ZM, Chen $\mathrm{X}, \mathrm{Gu} \mathrm{X}$, et al. A new magnetic resonance imaging scoring system for perilymphatic space appearance after intratympanic gadolinium injection, and its clinical application. J Laryngol Otol 2012;126:454-59

13. Baráth $\mathrm{K}$, Schuknecht B, Naldi AM, et al. Detection and grading of endolymphatic hydrops in Meniere disease using MR imaging. AJNR Am J Neuroradiol 2014;35:1387-92

14. Pyykko I, Nakashima T, Yoshida T, et al. Meniere's disease: a reappraisal supported by a variable latency of symptoms and the MRI visualisation of endolymphatic hydrops. BMJ Open 2013;3:e01555

15. Naganawa S, Satake H, Kawamura M, et al. Separate visualization of endolymphatic space, perilymphatic space and bone by a single pulse sequence: $3 \mathrm{D}$-inversion recovery imaging utilizing real reconstruction after intratympanic Gd-DTPA administration at $3 \mathrm{Te}$ sla. Eur Radiol 2008;18:920-24

16. Gürkov R, Flatz W, Louza J, et al. In vivo visualization of endolyphatic hydrops in patients with Meniere's disease: correlation with audiovestibular function. Eur Arch Otorhinolaryngol 2011;268:1743-48

17. Seo YJ, Kim J, Choi JY, et al. Visualization of endolymphatic hy- 
drops and correlation with audio-vestibular functional testing in patients with definite Meniere's disease. Audis Nasus Larynx 2013; 40:167-72

18. Fiorino F, Pizzini FB, Beltramello A, et al. Reliability of magnetic resonance imaging performed after intratympanic administration of gadolinium in the identification of endolymphatic hydrops in patients with Ménière's disease. Otol Neurotol 2011;32:472-77

19. Naganawa S, Yamazaki M, Kawai H, et al. Imaging of Ménière's disease by subtraction of MR cisternography from positive perilymph image. Magn Reson Med Sci 2012;11:303-09

20. Glockner JF, Hu HH, Stanley DW, et al. Parallel imaging: a user's guide. Radiographics 2005;25:1279-97

21. Moseley M, Liu C, Rodriguez S, et al. Advances in magnetic resonance neuroimaging. Neurol Clin 2009;27:1-19, xiii

22. Monsell EM. New and revised reporting guidelines from the Committee on Hearing and Equilibrium: American Academy of Otolaryngology-Head and Neck Surgery Foundation, Inc. Otolaryngol Head Neck Surg 1995;113:176-78

23. Mark AS, Seltzer S, Nelson-Drake J, et al. Labyrinthine enhancement on gadolinium-enhanced magnetic resonance imaging in sudden deafness and vertigo: correlation with audiologic and electronystagmographic studies. Ann Otol Rhinol Laryngol 1992;101:459-64

24. Claes G, Van den Hauwe L, Wuyts F, et al. Does intratympanic gadolinium injection predict efficacy of gentamicin partial chemolabyrinthectomy in Menière's disease patients? Eur Arch Otorhinolaryngol 2012;269:413-18

25. Fukuoka H, Takumi Y, Tsukada K, et al. Comparison of the diagnostic value of 3 T MRI after intratympanic injection of GBCA, electrocochleography, and the glycerol test in patients with Meniere's disease. Acta Otolaryngol 2012;132:141-45

26. Jerin C, Krause E, Ertl-Wagner B, et al. Longitudinal assessment of endolymphatic hydrops with contrast-enhanced magnetic resonance imaging of the labyrinth. Otol Neurotol 2014;35:880-83
27. Homann G, Fahrendorf D, Niederstadt T, et al. HR 3 Tesla MRI for the diagnosis of endolymphatic hydrops and differential diagnosis of inner ear tumors: demonstrated by two cases with similar symptoms. Rofo 2014;186:225-29

28. Naganawa S, Ishihara S, Iwano S, et al. Estimation of gadoliniuminduced T1-shortening with measurement of simple signal intensity ratio between the cochlea and brain parenchyma on 3D-FLAIR: correlation with T1 measurement by TI scout sequence. Magn Reson Med Sci 2010;9:17-22

29. Pyykko I, Zou J, Poe D, et al. Magnetic resonance imaging of the inner ear in Meniere's disease. Otolaryngol Clin N Am 2010;43 1059-80

30. Yoshioka M, Naganawa S, Sone M, et al. Individual differences in the permeability of the round window: evaluating the movement of intratympanic gadolinium into the inner ear. Otol Neurotol 2009; 30:645-48

31. Katayama N, Yamamoto M, Teranishi M, et al. Relationship between endolymphatic hydrops and vestibular-evoked myogenic potential. Acta Otolaryngol 2010;130:917-23

32. Tieleman A, Casselman JW, Somers T, et al. Imaging of intralabyrinthine schwannomas: a retrospective study of $\mathbf{5 2}$ cases with emphasis on lesion growth. AJNR Am J Neuroradiol 2008;29:898-905

33. Naganawa $S$, Satake H, Iwano $S$, et al. Communication between cochlear perilymph and cerebrospinal fluid through the cochlear modiolus visualized after intratympanic administration of Gd-DTPA. Radiat Med 2008;26:597-602

34. Kawai H, Naganawa S, Ishihara S, et al. MR imaging of the cochlear modiolus after intratympanic administration of Gd-DTPA. Magn Reson Med Sci 2010;9:23-29

35. Naganawa $S$, Ishihara $S$, Iwano $S$, et al. Detection of presumed hemorrhage in the ampullar endolymph of the semicircular canal: a case report. Magn Reson Med Sci 2009;8:187-91 